\title{
CRIANÇAS E ADOLESCENTES COM NECESSIDADES ESPECIAIS: DESAFIOS E MOTIVAÇÕES PARA CUIDADOS DE SAÚDE E EDUCAÇÃO ${ }^{1}$
}

\author{
CHILDREN AND ADOLESCENTS WITH SPECIAL NEEDS: \\ CHALLENGES AND MOTIVATIONS FOR HEALTH CARE AND EDUCATION
}

\author{
Andressa da Silveira ${ }^{2}$, Andréia Eckert Frank ${ }^{3}$, Gabriélli Maria Huppes ${ }^{3}$, \\ Luísa Pradié Algayer ${ }^{3}$, Gabrieli Beck Weide ${ }^{3}$ e Bruna da Rosa ${ }^{3}$
}

\section{RESUMO}

Este artigo objetiva descrever os desafios e as motivações de profissionais que atuam com crianças/adolescentes com necessidades especiais de saúde (CRIANES) que frequentam a clínica e a escola de uma Associação de Pais e Amigos dos Excepcionais (APAE). Trata-se de um estudo de abordagem qualitativa, do tipo participativo, onde a produção de dados ocorreu pelo desenvolvimento da dinâmica de criatividade e sensibilidade costurando estórias. Os participantes foram 13 profissionais da saúde e da educação, que estivessem atuando na APAE. As enunciações foram áudio gravadas, transcritas e submetidas à análise de discurso na corrente francesa. As expectativas em relação ao desenvolvimento das CRIANES perpetua no desejo dos profissionais, as atividades desenvolvidas são motivadas pelo afeto, carinho, amor, persistência e paciência. Para a equipe, o alcance da autonomia e das pequenas conquistas são essenciais para as crianças e adolescentes. Conclui-se que a valorização dos profissionais, as capacitações coletivas, o vínculo/participação das famílias na vida escolar e nos cuidados das CRIANES possibilitam melhorias significativas e maiores possibilidades para o desenvolvimento. Esse também é o desafio constante para os profissionais, visto que a assistência à saúde e a educação com CRIANES, transcendem limitações algumas vezes impostas por sua condição de saúde, outras pela sociedade.

Palavras-chave: Pessoas com Deficiência; Saúde da Criança; Saúde do Adolescente.

\section{ABSTRACT}

This article aims to describe the challenges and motivations of professionals who work with children/ adolescents with special health needs (CSHCN) who attend the clinic and school of an Association of Parents and Friends of Exceptional People (APAE). It is a qualitative study, of a participatory type, where the production of data occurred through the development of creativity and sensitivity dynamics by sewing stories. The participants were 13 health and education professionals, who were working at APAE. The statements were audio recorded, transcribed and subjected to discourse analysis in the French current. Expectations regarding the development of CSHCN perpetuate the professionals' desire, the activities developed are motivated by affection, affection, love, persistence and patience. For the team, the achievement of autonomy and small achievements are essential for children and adolescents. It is concluded that the valorization of the professionals, the collective qualifications, the bond / participation of the families in the school life and in the care of the CSHCN allow significant improvements and greater possibilities for development. This is also

\footnotetext{
${ }^{1}$ Artigo original com resultados parciais do projeto de pesquisa matricial "Tecnologias como possibilidades para o cuidado de crianças e adolescentes com necessidades especiais de saúde".

${ }^{2}$ Professora Coordenadora do Projeto de Pesquisa. Doutora em Enfermagem. Docente do Departamento de Ciências da Saúde, Universidade Federal de Santa Maria (UFSM), Campus de Palmeira das Missões. E-mail: andressadasilveira@gmail.com

${ }^{3}$ Estudantes do Curso de Graduação em Enfermagem, Universidade Federal de Santa Maria (UFSM), Campus de Palmeira das Missões. E-mails: andreiafrank93@gmail.com, gabriellihuppes@gmail.com, luisaapradie@gmail.com, gabrielibecker12@gmail.com, brunarosa317@gmail.com
} 
a constant challenge for professionals, since health care and education with CSHCN transcend limitations, sometimes imposed by their health condition, other times by society.

Keywords: Disabled Persons; Child Health; Adolescent Health.

\section{INTRODUÇÃO}

Na década de 90 ocorreram mudanças significativas na taxa de mortalidade infantil, por meio de avanços científico-tecnológicos na assistência à saúde pediátrica, sobrevivência de crianças e adolescentes considerados frágeis, necessidades de cuidados de saúde clinicamente complexos, que refletiram na sobrevida de crianças e adolescentes, que passaram a viver com demandas de cuidados especiais de saúde (SILVEIRA, NEVES; 2020).

Crianças e adolescentes com necessidades especiais de saúde (CRIANES) necessitam de cuidados de saúde, para além de cuidados cotidianos apresentados por outras crianças e adolescentes. As CRIANES caracterizam-se pela pluralidade de diagnósticos médicos, e recebem classificação de acordo com as demandas de cuidados apresentadas. Um instrumento de triagem desenvolvido nos Estados Unidos, apresenta seis grupos de demandas de saúde: uso de medicamentos contínuos, necessidade de utilizar serviços médicos, utilização de serviços de saúde mental ou psicopedagógicos, presença de limitação funcional, necessidade de terapia de reabilitação e tratamento ou aconselhamento para problemas emocionais, de desenvolvimento ou de comportamento (BETHELL et al., 2008).

Os profissionais que atuam no cuidado, assistência e educação de CRIANES necessitam de formação específica, a fim de que possam desenvolver cuidados contínuos em prol do desenvolvimento dessa população, realizar o acolhimento e ser comprometido para planejar ações que supram as necessidades de diferentes redes de apoio (VAZ et al., 2019).

Neste sentido, é fundamental articular saberes, complementando as diferentes áreas do saber, como saúde e educação, a fim de superar a visão fragmentada de necessidades de apoio e cuidado. O profissional que atua com crianças e adolescentes com necessidades especiais deve estar preparado para uma abordagem de cuidado integral, que supera a discriminação para conhecer sobre a história de vida, a dinâmica e formação familiar, os aspectos culturais, valores, situação financeira e fontes de apoio social (OTHERO, AYRES; 2012).

No que se refere as famílias de CRIANES, muitas não recebem suporte adequado, encontrando alguns obstáculos desde o diagnóstico até a rede de cuidados, desamparo na atenção primária, dificuldades de acesso e acessibilidade que podem fragilizar ainda mais a CRIANES, que tem o serviço hospitalar como porta de entrada para suas demandas de saúde (NEVES et al., 2019).

As CRIANES trazem de forma emergente, a necessidade de uma rede que garanta a integralidade do cuidado às pessoas que vivem com algum tipo de deficiência, ações e serviços articulados 
entre si, que favoreçam as parcerias entre os diversos serviços e atores da rede, comprometimento de trabalhadores e gestores em prol das necessidades de saúde dessa população (DUBOW, GARCIA, KRUG; 2018).

Entre as redes de apoio e segmentos profissionais que desenvolvem cuidado, assistência e educação, destacam-se aqueles que atuam na Associação de Pais e Amigos dos Excepcionais (APAE). Para Silveira et al. (2020), o nascimento da APAE contou com apoio de diversos segmentos da saúde, educação, assistência social e famílias, a fim de incluir as pessoas com deficiência e desenvolver uma atenção especial e qualificada. A APAE possibilita o acesso, a acessibilidade e a inclusão social, para que as CRIANES possam desenvolver suas potencialidades e habilidades, em prol da autonomia e melhoria das condições de saúde e aprendizado.

A partir dessas assertivas, questiona-se: "De que forma os profissionais da saúde e da educação desenvolvem seu trabalho com CRIANES?"

Este estudo tem por objetivo descrever os desafios e as motivações de profissionais que atuam com CRIANES que frequentam a clínica e a escola de uma APAE.

\section{MÉTODO}

Este artigo apresenta os resultados parciais do banco de dados de um projeto de pesquisa matricial "Tecnologias como possibilidades para o cuidado de crianças e adolescentes com necessidades especiais de saúde".

Trata-se de uma pesquisa de abordagem qualitativa, tipo participativa, em que a produção de dados ocorreu por meio do Método Criativo e Sensível (MCS), a partir da Dinâmica de Criatividade e Sensibilidade (DCS) “Costurando Estórias”.

O MCS possibilita que os participantes possam socializar suas vivências, por meio de reflexões e discussões coletivas. A DCS “Costurando Estórias”, utiliza como produção artística o entrelaçar de um novelo de linha, onde os participantes da DCS podem socializar, dialogar e apresentar problemas e dificuldades com raízes sociais e coletivas (SILVEIRA, et al., 2013). A produção de dados foi realizada no primeiro semestre de 2019, com profissionais das áreas da saúde e da educação, de uma APAE localizada no Sul do Brasil.

Foram inclusos na pesquisa profissionais que estivessem vinculados a equipe de trabalho da APAE há pelo menos seis meses, e que desenvolvessem atividades laborais com crianças e adolescentes. Aplicou-se como critério de exclusão, profissionais que estivessem afastados do trabalho devido a licença saúde ou férias.

No período da produção de dados, a equipe era composta por 17 profissionais da saúde e da educação, sendo que para a composição dos participantes do estudo aplicou-se os critérios de inclusão/exclusão. Deste modo, participaram da DCS 13 profissionais (pedagogos, terapeuta ocupacional, 
psicóloga, assistente social, professor de educação física, fisioterapeuta), que atuavam diretamente com crianças e adolescentes na APAE.

Para a operacionalização da DCS, inicialmente foi realizada a apresentação do Projeto para a direção da APAE, posteriormente foi realizado o convite para os profissionais que correspondiam aos critérios do estudo, não havendo recusas. No dia seguinte, uma sala foi reservada para a atividade, com espaço amplo, onde foram disponibilizadas as cadeiras em formato circular. Entre os materiais utilizados para a DCS destaca-se o novelo de linha, crachás, folhas e canetas para a identificação dos participantes. Na lousa foi inclusa a questão geradora de debate que conduziu a DCS: "Como você realiza seu trabalho com crianças e adolescentes que possuem necessidades especiais de saúde?”.

A produção de dados seguiu as seguintes etapas: recepção/acolhimento dos participantes, apresentação individual de cada participante, introdução do objetivo do estudo e da questão geradora de debate, disponibilização de materiais, realização da DCS com a socialização de ideias e reflexões, validação e finalização da dinâmica.

As enunciações dos participantes foram gravadas em mídia digital, transcritas e submetidas à Análise de Discurso (AD) na corrente francesa. Para a $\mathrm{AD}$ seguiu-se as seguintes etapas: dupla transcrição dos dados empíricos, materialidade linguística, extração e a organização do discurso em quadros analíticos e por fim, a categorização (SILVEIRA, SOUZA, SILVA; 2019).

Utilizou-se as ferramentas analíticas: metáfora, paráfrase e polissemia. Além disso, aplicou-se os recursos ortográficos do Programa Microsoft Word, que possibilitaram dar movimento aos discursos dos participantes, por meio da legenda: / pausa curta; // pausa longa; // pausa muito longa; ... pensamento incompleto; \# interrupção da enunciação; [ ] para inserir informação completar ao enunciado; “...” aspas duplas indicam a fala ou texto de outrem reproduzidos pelo entrevistado; [...] indica que houve um corte na fala.

O projeto de pesquisa foi cadastrado no portal de projetos da Universidade Federal de Santa Maria, posteriormente na Plataforma Brasil e obteve a aprovação do Comitê de Ética e Pesquisa em 2018, com parecer n ${ }^{\circ}$ 2.62.767. A pesquisa seguiu as diretrizes estabelecidas na Resolução 466/12, que institui as normas para o desenvolvimento de pesquisas com seres humanos. Para sigilo da identidade, utilizou-se a letra "P" referente a participante, acompanhada por número ordinal aleatório, da seguinte forma: P1, P2, P3, ..., P13.

\section{RESULTADOS}

Os discursos dos profissionais da saúde e da educação enaltecem sobre os sentimentos, que os motivam a continuar na APAE e a acreditar nas possibilidades de desenvolvimento das CRIANES, com destaque para o amor às crianças e adolescentes. 
[...] Mas essa parte do amor, se a gente quer ser amado de verdade, a gente vem trabalhar com eles! Eu me realizo! Eu passei por momentos difíceis na minha vida, mas aqui eu encontrei o apoio, o amor que eu precisava... [...](P3)

[...] Se a gente for pensar... // o que rege toda história da humanidade é o amor... Faz sentido na nossa vida, faz sentido no nosso trabalho principalmente, trabalhar com amor... (P4)

[...] Nós devemos ter amor em tudo que nós fizermos! Tudo que a gente for fazer, a gente tem que botar amor no que faz! Acho que é essencial... (P6)

É assim, bem gratificante! E aqui, eu fui e estou sendo bem-amada! (P7)

Além disso, as enunciações trazem as motivações que amparam os profissionais da saúde e da educação, mesmo diante das limitações do cotidiano, fortalecendo sobre a importância de manter a persistência, a esperança e o respeito.

[...] Às vezes, claro, que a gente se abala...// Por que a gente é humano além de profissional, mas com persistência vamos lá! (Pl)

[...] A felicidade também está nas pessoas, nos colegas, nos alunos, também nos momentos bons que a gente vive! (P8)

O que nos motiva / é a esperança /l, que nos motiva todos os dias, pra gente persistir... [...] (P9)

[...] É bem importante... // a gente respeitar os outros, nossos alunos, usuários também! Por que eles também têm o direito de estar aqui! (P12)

Os participantes apresentam em seus movimentos discursivos, a função do profissional da saúde e da educação na APAE. Em relação ao papel da equipe, os desafios em desenvolver as atividades laborais com CRIANES e suas possibilidades, com ênfase na coletividade, no engajamento familiar por meio da atenção e comunicação.

Tu está sempre em cima, procurando preservar o melhor para eles, mas a gente tem que dar amor para eles, atenção para eles, é isso que é importante! Mas também é desafiador! (P2)

Afeta bastante a todos, / principalmente a familia envolvida, porque a gente não trata apenas a criança e o adolescente, / mas todos os aspectos envolvidos... [...] (P5)

[...] Sempre tento focar no bem-estar e felicidade das crianças e adolescentes, conversando com eles! (P10)

Eu procuro dar o melhor de mim, \# tanto para alunos, como para usuários e famílias... [...] procuro atender as necessidades de cada um ... [...] (P11)

[...] Informação, atendimento que elas não têm na rede. Como a APAE é uma instituição que oferece esse conjunto completo na parte da saúde, na parte da escola uma educação especializada... [...] Contempla os direitos, a educação e a saúde... (P13) 
No que se refere a realização profissional, os discursos são dicotômicos. Percebe-se que para alguns, o começo de suas atividades laborais era permeado pela vontade de transformar a realidade das CRIANES. Todavia, com o passar do tempo, as motivações transformaram-se em desafios para a continuidade do trabalho.

Sempre que a gente chega, // a gente se frustra um monte! A gente acha que mesmo sendo com as pessoas especiais, a gente vai conseguir mudar alguma coisa, dai a gente vai trabalhando e vai refletindo... [...] (P3)

[...] Tem algumas dificuldades, que muitas vezes, tem que explicar tudo novamente, tem que ser bem explicadinho... (P5)

[...] É muito frustrante! Quando eu vim para cá eu tinha uma expectativa, e não é a expectativa que eu esperava, eu encontrei muitos desafios na aprendizagem... [...] (P6)

Quando eu vim para cá eu tinha uma realidade, // não é aquilo que tu sonha, pois na verdade a gente quer dar asas para eles voarem... (P10)

Os discursos dos profissionais remetem sobre a importância de querer ir além, de fazer algo a mais, e de construir possibilidades, mesmo diante das limitações impostas pelas necessidades de saúde e educação.

[...] Penso sempre no que eu posso fazer, qual é a minha postura, o que eu posso tentar realizar para ajudar de alguma forma... (P9)

[...] Te passa mil e uma atividades na cabeça, mas não consegue realizar aquela atividade... // Mas mesmo assim, acho que a gente não pode desanimar! (P10)

A realização pode ser uma forma de motivação para a gente, / a partir do momento que você está realizado pessoalmente ou profissionalmente... É sempre uma forma de você procurar um objetivo maior / e sempre fazer o melhor! (P13)

[...] Acho que é isso, o que a gente pode estar oferecendo para eles... [...] Cada um, na sua forma, tenta oferecer o melhor de si! (P14)

Sobre a participação familiar, denota-se que por um lado as famílias desejam que as mudanças e o processo de desenvolvimento de CRIANES seja rápido. Por outro lado, o profissional da saúde e da educação compreende que requer tempo, que cada criança e adolescente possui seu tempo. Os discursos enfatizam ainda, a necessidade de participação familiar, e a importância da troca de saberes.

[...] Tem uns que começam a ficar críticos, a saber mais das coisas e \# se interessam mais, sabem mais da realidade, pois se interessam mais... (P2)

É assim, a gente queria muito que essas famílias entendessem, // que isso aqui [APAE], é uma escola de atendimento especial. Eles precisam do acompanhamento e do comprometimento, dos pais e da família! (P4) 
[...] As famílias trazem para a APAE, / sempre é uma troca, é uma palavrinha, sabe? Aí você vê a realização do seu trabalho. As familias percebem o trabalho e valorizam muito... \# (P9)

Os pais querem que aconteça os avanços das crianças e adolescentes, e a gente sabe que não vai ser tão rápido, / mas eu acho que a gente afeta quase que diretamente a família $\operatorname{assim\ldots }(P 13)$

Metaforicamente, algumas enunciações apresentam o engajamento dos profissionais por meio de falas, como "vestir a camiseta" e "correr atrás". Quanto a equipe de saúde e da educação, alguns discursos apresentam a necessidade de inserção do enfermeiro na APAE, as competências profissionais de cada área, mérito pelos esforços coletivos e as diferenças entre a avaliação de desenvolvimento clínico e escolar.

Tem que ter as pessoas com vontade de trabalhar, que vistam a camiseta da APAE! O profissional deve querer fazer algo diferente! (P2)

A escola não ampara grupo de convivência. Esse grupo de convivência a escola não pode dar, o grupo de convivência é da assistência! (P4)

Até um enfermeiro fazer parte! / Por segurança, por ter alguns cuidados que outras áreas já não dominam, cada um tem a sua parte de cuidado. [...] A maior preocupação com a questão da inclusão das pessoas com deficiência.......] (P5)

[...] Alguns alunos nossos vão em alguns programas da assistência que eles participam, mas é muito pouco, acho que tem uns três apenas... (P9)

Na parte clínica, / a alta é de acordo com cada um, o que cada setor almeja. O limite na parte da educação intelectual já pesquisei, já fui atrás, e eles estão formulando agora uma disciplina obrigatória. Tem que ir atrás! (P14)

Os discursos dos participantes reforça sobre a necessidade de inclusão das CRIANES em outros espaços de convivência, sobre o papel dos profissionais de saúde e educação, o vínculo construído, e a constante busca para a autonomia e desenvolvimento das crianças e adolescentes. Desta forma, as práticas de assistência, cuidado e educação fazem parte do cotidiano de trabalho desses profissionais.

\section{DISCUSSÃO}

As CRIANES representam um grupo que requer cuidados específicos e diferenciados de saúde (MONNERAT et al., 2016). Os cuidados podem ser temporários ou permanentes, dependem da continuamente dos serviços de saúde e de diferentes profissionais que fazem acompanhamento. Para Nogueira Reis et al. (2017), a qualidade de vida das CRIANES relaciona-se também com os serviços de saúde e redes de apoio, reorganizações familiares e cuidados específicos às necessidades de saúde dessa população. 
Para atuar com este público é necessário que o profissional tenha estabilidade emocional, inclusive por sentirem-se comovidos, abalados e fragilizados em algumas situações (ROSSO, LOSSO; 2016). Os laços de afeto entre paciente e cuidador, transforma-se em compartilhamento de vivências, carinho e amizade. Algumas vezes, precisa ser trabalhada a motivação para a continuidade dos cuidados e os desafios que fazem parte do cotidiano de uma CRIANES (CRUZ et al., 2017).

As formações discursivas remetem sobre as dificuldades de aprendizado, e o trabalho contínuo por tempo indeterminado. Alguns discursos denotam sobre os desafios e até mesmo frustrações, diante das incertezas em desenvolver as atividades planejadas (VIANA et al., 2018). O processo de aprendizagem resulta de uma construção pessoal extremamente complexa, envolvendo aspectos cognitivos, emocionais, orgânicos, psicossociais e culturais (TABILE, JACOMETO; 2017).

No que tange ao cuidado compartilhado com a família, o profissional torna-se próximo da família e da CRIANES, sua atuação vai além da capacidade de desenvolver procedimentos técnicos especializados, mas envolve o vínculo, a troca de saberes e a educação em saúde com os cuidadores familiares (CRUZ et al., 2017). Diante das demandas de cuidados da CRIANES, os familiares podem sofrer mudanças drásticas em seu cotidiano, frente a necessidade de atenção integral e cuidados complexos. Neste sentido, cabe a equipe multiprofissional ofertar apoio aos cuidadores, amenizando os sentimentos negativos e de desemparo, contribuindo para superação dos desafios (NOGUEIRA REIS et al., 2017).

Já no aspecto de ensino e de aprendizagem, a educação deve ser capaz de transcender limitações da aprendizagem e identificar as possibilidades da CRIANES envolvendo a família neste processo (TABILE, JACOMETO; 2017). Os profissionais da saúde e da educação percebem a importância do trabalho que desenvolvem com as CRIANES usuárias da APAE. Para eles, o processo de desenvolvimento, de aquisição de habilidades e adesão ao tratamento dependem do vínculo com as famílias, de esforços compartilhados entre clínica, escola e família (SILVEIRA et al., 2020). Para o êxito no processo de desenvolvimento das CRIANES é crucial que o profissional tenha formação específica para trabalhar com esta população, e que seja capaz de dialogar com os familiares a respeito das demandas apresentadas pelas crianças e adolescentes (INÁCIO, PEIXOTO; 2017).

Além disso, salienta-se a importância das redes de apoio acessadas por CRIANES e suas famílias, a fim de que haja continuidade dos cuidados de saúde e educação especializada. A APAE desenvolve essa articulação da CRIANES com a assistência e cuidado, contempla a educação especializada e ainda, a socialização das crianças e adolescentes usuárias. De acordo com Silveira, Neves e Paula (2013), a escuta sensível, as possibilidades para a manutenção do cuidado em casa, e as redes de apoio social ou institucional são fontes promotoras para o empoderamento, desenvolvimento e inclusão da criança ou adolescente.

A troca de saberes e o vínculo, entre a equipe da APAE e as famílias de CRIANES podem reduzir os fatores que dificultam a adesão ao tratamento (SILVEIRA et al., 2020). A CRIANES necessita de estratégias que tenham o objetivo de melhorar sua qualidade de vida e o bem-estar (INÁCIO, 
PEIXOTO; 2017). Com intuito de projetar o plano de cuidados, de acordo com o contexto familiar em que a CRIANES está inserida(DIAS, ARRUDA, MARCON; 2017).

Para os profissionais que atuam na APAE, as CRIANES e suas famílias buscam na instituição apoio para as demandas de cuidados, educação especializada, socialização e suporte emocional. O processo de desenvolvimento, de aquisição de habilidades e adesão ao tratamento precisam de esforços coletivos da clínica, da escola e das famílias (SILVEIRA et al., 2020). Ressalta-se ainda, o vínculo empático, longitudinal e integral entre a equipe multiprofissional e os cuidadores familiares de CRIANES, visto que o familiar desempenha o exercício diário do cuidado e a busca incessante pela inserção na rede de atenção à saúde e intersetorial (DIAS, ARRUDA, MARCON; 2017).

Os participantes enalteceram sobre a importância da inserção do enfermeiro na APAE, visto que a ausência da enfermagem faz com que a equipe busque suporte em outros espaços de saúde. Para Cabral e Moares (2015) é necessário ampliar a visibilidade dos cuidados de enfermagem, sobretudo no contexto das demandas de cuidados requeridos pelas CRIANES, fortalecendo a enfermagem como parte da rede de cuidados dessa população. Assim, a introdução e a participação da enfermagem em espaços que possuem pessoas com necessidades especiais é imprescindível para a promoção da saúde e melhoria da qualidade de vida.

\section{CONCLUSÃO}

A APAE além de prestar assistência social, de saúde e suporte educacional especializado, é um espaço de socialização e integração das CRIANES. Esforços coletivos, persistência e participação familiar podem refletir de forma positiva para autonomia e desenvolvimento da CRIANES.

A valorização dos profissionais da saúde e educação, a afetividade, o vínculo e a participação das famílias são fontes motivacionais. Já a singularidade de cada CRIANES, o tempo indeterminado para a aquisição de habilidades, desenvolvimento e autonomia são os maiores desafios.

Constatou-se a ausência da enfermagem entre os profissionais da saúde que atuam na APAE. Todavia, a presença do enfermeiro mostra-se favorável, a fim de desenvolver um plano de cuidados e educação em saúde, com a CRIANES e sua família.

Por fim, recomenda-se a utilização de estudos que trazem metodologias participativas, a possibilidade de trocas de saberes e reflexão coletiva para temas sensíveis.

A valorização dos profissionais, as capacitações coletivas, o vínculo/participação das famílias na vida escolar e nos cuidados das CRIANES possibilitam melhorias significativas, e maiores possibilidades para o desenvolvimento. Esse também é o desafio constante para os profissionais, visto que a assistência à saúde e a educação com CRIANES transcendem limitações, algumas vezes impostas pela condição de saúde, outras pela sociedade. 
A respeito das limitações deste estudo, a produção de dados foi realizada no momento em que alguns profissionais estavam afastados das atividades laborais, contemplando parcialmente a equipe da APAE.

\section{REFERÊNCIAS}

BETHELL, C.D. et al. What is the prevalence of children with special health care needs? Toward an understaulnding of variations in findings and methods across three national surveys. Maternal and Child Health Journal, v. 12, n. 1, p. 1-14, 2008. Disponível em: https://bit.ly/32u9ACv. Acesso em: jul. 2020

CABRAL, I.E.; MORAES, J.R.M.M. Cuidadores familiares articulando a rede social de uma criança com necessidades especiais de saúde. Revista Brasileira de Enfermagem, Brasília, v. 68, n. 6, p. 1078-1085, 2015. Disponível em: https://bit.ly/3kpINNT. Acesso em: jul. 2020.

CRUZ, C.T. et al. Atenção à criança com necessidades especiais de cuidados contínuos e complexos: percepção da enfermagem. Revista Mineira de Enfermagem, 21:e-1005, 2017. Disponível em: https://bit.ly/2Ud10n0. Acesso em: fev. 2020.

DIAS, B.C.; ARRUDA, G.O.; MARCON, S.S. Vulnerabilidade familiar de crianças com necessidades especiais de cuidados múltiplos, complexos e contínuos. Revista Mineira de Enfermagem, 21:e-1027, 2017. Disponível em: https://bit.ly/35lgNXr. Acesso em: jan. 2020.

DUBOW, C.; GARCIA, E.L.; KRUG, S.B.F. Percepções sobre a Rede de Cuidados à Pessoa com Deficiência em uma Região de Saúde. Saúde em debate, Rio de Janeiro, v. 42, n. 117, p. 455-467, 2018. Disponível em: https://bit.ly/3naoQvZ. Acesso em: jul. 2020.

INÁCIO; A.L.R.; PEIXOTO, A.P.G.L. A assistência de enfermagem e o cuidado familiar às crianças com necessidades especiais de saúde: uma revisão integrativa. Revista de Atenção à Saúde, São Caetano do Sul, v. 15, n. 53, p. 87-94, 2017. Disponível em: https://bit.ly/2UeU1J8. Acesso em: jan. 2020.

MONNERAT, C.P. et al. Estratégia de educação em saúde com familiares de crianças em uso contínuo de medicamentos. Revista de Enfermagem UFPE on line, v. 10, n. 11, p. 3814-22, 2016. Disponível em: https://bit.ly/3eLdmfJ. Acesso em: jan. 2020.

NEVES, E.T. et al. Acesso de crianças com necessidades especiais de saúde à rede de atenção. Revista Brasileira de Enfermagem, Brasília, v. 72, supl. 3, p. 65-71, 2019. Disponível em: https://bit.ly/36h6DGo. Acesso em: jul. 2020. 
NOGUEIRA REIS, K.M. et al. A vivência da família no cuidado domiciliar à criança com necessidades especiais de saúde. Ciencia y enfermería, Concepción, v. 23, n. 1, p. 45-55, 2017. Disponível em: https://bit.ly/3114mA8. Acesso em: jul. 2020.

OTHERO, M.B.; AYRES, J.R.C.M. Necessidades de saúde da pessoa com deficiência: a perspectiva dos sujeitos por meio de histórias de vida. Interface - Comunicação, Saúde, Educação, Botucatu, v. 16, n. 40, p. 219-234, 2012. Disponível em: https://bit.ly/3nnbHA7. Acesso em: jul. 2020.

ROSSO, L.E.; LOSSO, A.R.S. Cuidado de enfermagem na APAE: necessidades da equipe multiprofissional. Revista Inova Saúde, Criciúma, v. 5, n. 2, 2016. Disponível em: https://bit.ly/2IiRVGy. Acesso em: jul. 2020.

SILVEIRA, A.; NEVES, E.T. Adolescentes com necessidades especiais de saúde no Brasil: demandas de cuidado. In: Associação Brasileira de Enfermagem, Sociedade Brasileira de Enfermeiros Pediatras. Programa de Atualização em Enfermagem - PROENF Saúde da criança e do Adolescente. Ciclo 14, v.4. Porto Alegre: Artmed Panamericana, 2020. p. 141-66.

SILVEIRA, A. et al. Participação e ausência familiar: implicações para o desenvolvimento de crianças e adolescentes com necessidades especiais. Revista Contexto \& Saúde, Ijuí, v. 20, n. 38, p. 185-190, 2020. Disponível em: https://bit.ly/3kcPD93. Acesso em: jun. 2020.

SILVEIRA, A.; SOUZA, N.S; SILVA, E.B. Análise de discurso francesa: relato sobre sua aplicabilidade em estudos de enfermagem. In: $O$ conhecimento na competência da teoria e da prática em enfermagem, v. 2. Ponta Grossa: Atena Editora, 2019. p.90-97.

SILVEIRA, A. et al. Educação em saúde: saberes dos familiares de crianças com necessidades especiais de saúde. Revista de Enfermagem UFPE on line, Recife, 7(esp):6190-6196, 2013. Disponível em: https://bit.ly/2JVvoQX. Acesso em: jul. 2020.

SILVEIRA, A.; NEVES, E.T.; PAULA, C.C. Cuidado familial das crianças com necessidades especiais de saúde: um processo (sobre)natural e de (super)proteção. Texto \& Contexto Enfermagem, Florianópolis, v. 22, n. 4, p. 1106-1114, 2013. Disponível em: https://bit.ly/3eKlyfX. Acesso em: jul. 2020.

TABILE, A.F.; JACOMETO, M.C.D. Fatores influenciadores no processo de aprendizagem: um estudo de caso. Revista Psicopedagogia, São Paulo, v.34, n.103, p.75-86, 2017. Disponível em: https://bit.ly/ 3eNRu3k. Acesso em: jul. 2020. 
VAZ, E. M. C. et al. Coordenação do cuidado na Atenção à Saúde à(ao) criança/adolescente em condição crônica. Revista Brasileira de Enfermagem, Brasília, v. 71, supl. 6, p. 2612-2619, 2018. Disponível em: https://bit.ly/3eIuAKu. Acesso em: jan. 2020.

VIANA, I. S. et al. Encontro educativo da enfermagem e da família de crianças com necessidades especiais de saúde. Texto \& Contexto Enfermagem, Florianópolis, v. 27, n. 3, e5720016, 2018. Disponível em: https://bit.ly/35iJHaB. Acesso em: jan. 2020. 